\title{
Immigration, croissance démographique et dynamique urbaine au Nord Cameroun
}

\author{
Félix Watang Zieba \\ Département de Géographie, ENS, Université de Maroua, \\ BP 55 Maroua, +237 995391 98, \\ watangf@yahoo.fr
}

\begin{abstract}
The emergence of towns with millions of people like Yaounde and Douala in Cameroon is a rapid manifestation of the recent urban dynamics taking place in the countries of the south. These towns which are located in the southern part of the country benefitted from the influx of population from other regions of the country up to the early 1980's. The north of Cameroon which is the most populated part of the country was a departure point to the towns of the south to the detriment of the towns of the north. Nevertheless there was a rapid development of the major towns of this part of the territory from the end of the 1980 decade. Surveys were carried out on the migrants' communities and the local authorities in the three major towns of the north (Maroua, Garoua, Ngaoundere) and in the secondary towns of Kousseri, Guider and Figuil to identify the countries of origin of the migrants and their role in urban dynamics. Data from the recent population census was also used. Statistical, cartographic and qualitative analysis of demographic data from field survey and the national population and housing census (RGPH) of 1987 and 2005, brings out an urban development phenomenon in this region on both a demographical and spatio economical view point in relation with the internationalisation of north Cameroon migratory field.
\end{abstract}

Keywords:urban dynamics;transfrontier and internal migration; North Cameroon

\section{Résumé}

La dynamique urbaine récente qui touche les pays du sud s'est manifestée rapidement au Cameroun par l'émergence des villes millionaires que sont Yaoundé et Douala. Ces villes situéesdans la partie méridionale du pays ont bénéficié jusqu'au début des années 1980, de l'essentiel du flux des populations venant de toutes les autres régions du pays. La partie septentrionale, espace le plus peuplé du pays était alors considéréecomme point de depart vers les villes méridionales au détriment des villes septentrionales. Cependant à partir de la fin de la décennie 1980, on notera un développement rapide des principales villes de cette partie du territoire. Des enquêtes ont été menéesdans les trios principales villes septentrionales (Maroua, Garoua, Ngaoundéré) et dans les villes secondaires de Kousséri, Guider, Figuil auprès des communautés des immigrés et des autorités locales. Il s'est agit d'identifier les pays d'origine des immigrés et leur role dans la dynamique urbaine. Les données issues des different recensements de la population ont aussi été utilisées. Les analyses statistique, cartographique et qualitative des données démographiques issues des enquêtes de terrain et des recensements généraux de la population et de l'habitat (RGPH) de 1987 et 2005, font apparoir un développement du phenomena urbain dans cette region tant du point de vue démographiqueque spatio-économique en relation avec l'internationalisation du champ migratoire nord camerounais.

Mots clés: dynamique, urbaine, migrations transfrontalières et internes, Nord Cameroun

\section{Introduction}

Depuis les années 1960, la polarisation de l'espace camerounais fut marquée par deux pôles urbains (Yaoundé et Douala) accueillant l'essentiel des migrants originaires des différentes régions du
Cameroun et même de la sous région Afrique centrale (Marguerat 1974; Dongmo, 1980). Si ces deux villes constituent toujours les principaux centres urbains du Cameroun, il n'en demeure pas moins que l'arrière pays longtemps considéré comme zone d'émigration se caractérise aujourd'hui 
par le développement du phénomène urbain. Dans la partie septentrionale en particulier à partir de 1983, la mise en place de nouveaux chefs lieux de province, le développement du transport et l'évolution des libertés individuelles va favoriser le développement des villes locales (Simeu Kamdem, 1985, 2000). C'est ainsi qu'en plus de la ville de Garoua, chef lieu de l'ancienne vaste province du Nord, vont s'ajouter les chefs lieux des deux nouvelles provinces (Maroua et Ngaoundéré) qui bénéficient dès 1983 d'une immigration sans précédente et participent à l'organisation spatiale et économique de leurs régions respectives. Ces villes vont connaître un développement rapide à partir des années 1990 à cause de l'accélération de l'exode rural, les campagnes étant les victimes directes de la chute des cours des produits de rente. En effet, la chute des cours du coton sera à l'origine de l'abandon de cette culture par les paysans qui la considèrent comme un pari perdu d'avance (Kossoumna et Havard, 2006 ; Watang Ziéba, 2010). La population de la ville de Maroua par exemple a doublé en six ans entre 1999 et 2005, elle est passée de 162000 à 300794 habitants, soit une croissance annuelle moyenne de $9 \%$. La population urbaine du Nord Cameroun en général a atteint près de deux millions en 2010 (RGPH, 2005), soit $20 \%$ de la population urbaine nationale. L'objectif de cet article est de caractériser ce phénomène sans cesse croissant et de déterminer les facteurs qui en sont responsables. II est structuré autour des questions suivantes: quels sont les indicateurs de l'urbanisation rapide du Nord Cameroun durant ces vingt dernières années? Quels sont les facteurs responsables de l'évolution du phénomène urbain dans cette partie du Cameroun? II s'appuie également sur l'hypothèse que la mobilité croissante des populations rurales vers les centres urbains proches, l'immigration transfrontalière sont responsables du développement des villes du Nord Cameroun ces vingt dernières années.

\section{Revue de la littérature et cadre théorique}

En 1950, la population urbaine mondiale était de 750 millions. En 1995, elle dépassait le cap de 2,5 milliards (ONU, 2010). En 2025, la population urbaine devrait constituée $80 \%$ de la population européenne et plus de la moitié de la population africaine (Wackermann, 2000). D'ailleurs en 2005, la population urbaine équivalait déjà à la moitié de la population mondiale (UNFPA, 20I2). Cette accélération du phénomène urbain est liée à plusieurs facteurs. L'un des facteurs les plus importants est l'exode rural qui a accompagné l'industrialisation dans les pays européens et la modernisation de l'économie dans les anciens pays colonisés. Pour Milhaud (1960), l'urbanisation « fait intégralement partie des transformations industrielles, culturelles et économiques du monde ". Les travaux des économistes comme Lewis (1954), Fei et Ranis, (1964) à travers le modèle d'économie dualiste arrivent à montrer que l'urbanisation est une conséquence de l'attraction des populations rurales par les centres industrialisés. II s'agit donc d'une des manifestations humaines de l'industrialisation. Le rapport entre l'urbanisation et l'immigration est bien présenté par Todaro (1969), Harris et Todaro (1970) qui insistent sur l'espérance de l'amélioration des revenus comme facteur sous-tendant les migrations des populations des zones rurales vers les zones urbaines. La croissance naturelle, le développement économique et l'aménagement du territoire (délocalisations des capitales, création de nouvelles villes) constituent des facteurs non négligeables de l'urbanisation, notamment dans les pays du sud. Pour Chaline (2000), les transformations des villes dépendent à la fois des facteurs exogènes dont les courants migratoires traduisent bien l'importance et d'une dynamique interne qui s'exprime notamment par la mobilité résidentielle et par les changements progressifs dans l'occupation sociale de l'espace intra-urbain. C'est dire que les mobilités géographiques sont au centre de la dynamique urbaine. Tel est aussi le point de vue de Guilmoto et Sandron (2000) qui considèrent "l'urbanisation comme la première des migrations ", établissant ainsi un lien fort entre ces deux phénomènes. Car l'urbanisation de l'Afrique est loin de s'achever à cause d'une part considérable de la population rurale qui ira s'installer dans les villes.

Le débat théorique autour du rapport existant entre les migrations et l'urbanisation en Afrique insiste sur l'inadéquation du modèle dualiste développé par Lewis (1954), Fei and Ranis (1964) dans un contexte différent marqué par une forte industrialisation urbaine ayant eu pour conséquence l'attrait massif des ruraux. D'abord lente dans les années 1960, l'exode rural en Afrique va s'accélérer deux décennies plus tard aussi bien à cause de la destruction des économies rurales (Courade 1994) que par l'espoir d'avoir un emploi en ville (Todaro 1969; Park and al. 2003). Cette évolution paraît d'ailleurs paradoxale parce qu'elle n'est la résultante d'aucune révolution industrielle comme ce fût le cas en Europe (Beauchemin et Bocquier 2004). Une prise en compte de l'émergence du secteur informel 
qui occupe la majorité des urbains et de la dégradation des conditions de vie dans les campagnes, permet de comprendre la forte urbanisation de l'Afrique subsaharienne malgré la morosité du secteur formel. Les capitales et les métropoles côtières sont considérées comme les principaux pôles d'attraction de l'essentiel des populations venant de l'hinterland (Dongmo, 1980 ; Gazel, op.cit.).

Dans le contexte camerounais, la région septentrionale est considérée comme zone d'émigration vers les pôles politiques et économiques de Yaoundé et de Douala. D'ailleurs, l'essentielle de la littérature sur les migrations au Nord-Cameroun insiste plus sur le peuplement récent des espaces ruraux de faible densité (Roupsard, 1987; Beauvilain, 1989; Ndembou, 2010), sur l'émigration vers les bassins sucriers méridionaux (Zoa, 20l0) et vers les grandes villes méridionales (Gubry et al., 199I, 1995). Malgré l'internationalisation du champ migratoire de cette partie du Cameroun suite à l'immigration tchadienne, nigériane, nigérienne, centrafricaine et même malienne, le lien entre migration internationale et urbanisation au Nord Cameroun n'a pas eu des chercheurs l'attention qu'il mérite. Les études urbaines se sont limitées ici à la caractérisation de l'extension spatiale des villes de Garoua et Maroua, à l'analyse de la question foncière urbaine (Seignobos, 2000 ; Simeu Kamdem, 2000).

\section{Cadre conceptuel}

Les concepts fondamentaux qui sous-tendent cette étude sont ville, urbanisation et immigration.

La ville est définie par Georges et Verger (1996) comme un groupement de population agglomérée défini par un effectif de population et par une forme d'organisation économique et sociale. Le critère démographique apparaît essentiel car dans plusieurs pays, un effectif standard est fixé en dessous duquel une agglomération ne peut être considérée comme ville. Pour les Nations Unies, est considérée comme ville toute agglomération d'au moins 100000 habitants. Au Cameroun, ce chiffre est fixé à 5000 habitants. Dans le cadre de cette étude seules les agglomérations ayant au moins 20000 habitants ont été prises en compte. Le processus de développement des villes en nombre d'habitants, en extension territoriale peut être considéré comme urbanisation ou dynamique urbaine. Bien que pas exclusif, le caractère démographique de la dynamique urbaine a été privilégié dans cette étude. Les effets spatiaux de l'extension du champ migratoire nord camerounais constituent une autre caractéristique de la dynamique urbaine étudiée. II s'agit de prendre en compte l'extension spatiale des principales villes étudiées et le développement des petits centres à vocation agricole longtemps considérés comme des gros villages. Des études et programmes de recherche portant sur la dynamique urbaine dans le monde en général et en Afrique en particulier ont parfois privilégié la première approche (Le Bris, 200I ; ONU, 2010) ou concilié les deux (Gazel et al., 20l0). Cette deuxième option convient bien à cette étude où il est question de mettre en rapport l'immigration et la dynamique urbaine. L'immigration qui est considérée comme un changement de résidence d'un certain lieu de départ à un lieu d'arrivée (Noin, 1995) prend ici en compte l'exode rural, la mobilité interurbaine et l'immigration internationale.

\section{Données et méthode:}

\section{La zone d'étude}

Cette recherche s'appuie du point de vue spatial sur le Nord Cameroun en général. Cette partie du pays est constitué de trois régions administratives (Extrême-Nord, Nord et Adamaoua) (figure I) et s'étend en latitude sur $7^{\circ}\left(\mathrm{N6}^{\circ}-\mathrm{N} 13^{\circ}\right)$ et en longitude sur $5^{\circ}\left(\mathrm{E} 10^{\circ} 30^{\prime}-\mathrm{EI} 5^{\circ}\right)$. Elle est caractérisée du sud vers le nord par les climats soudaniens d'altitude (Adamaoua), soudano-sahélien (Nord) et sahélien (Extrême-Nord). Sa population est de 5684040 habitants, soit $33 \%$ de la population du Cameroun. 


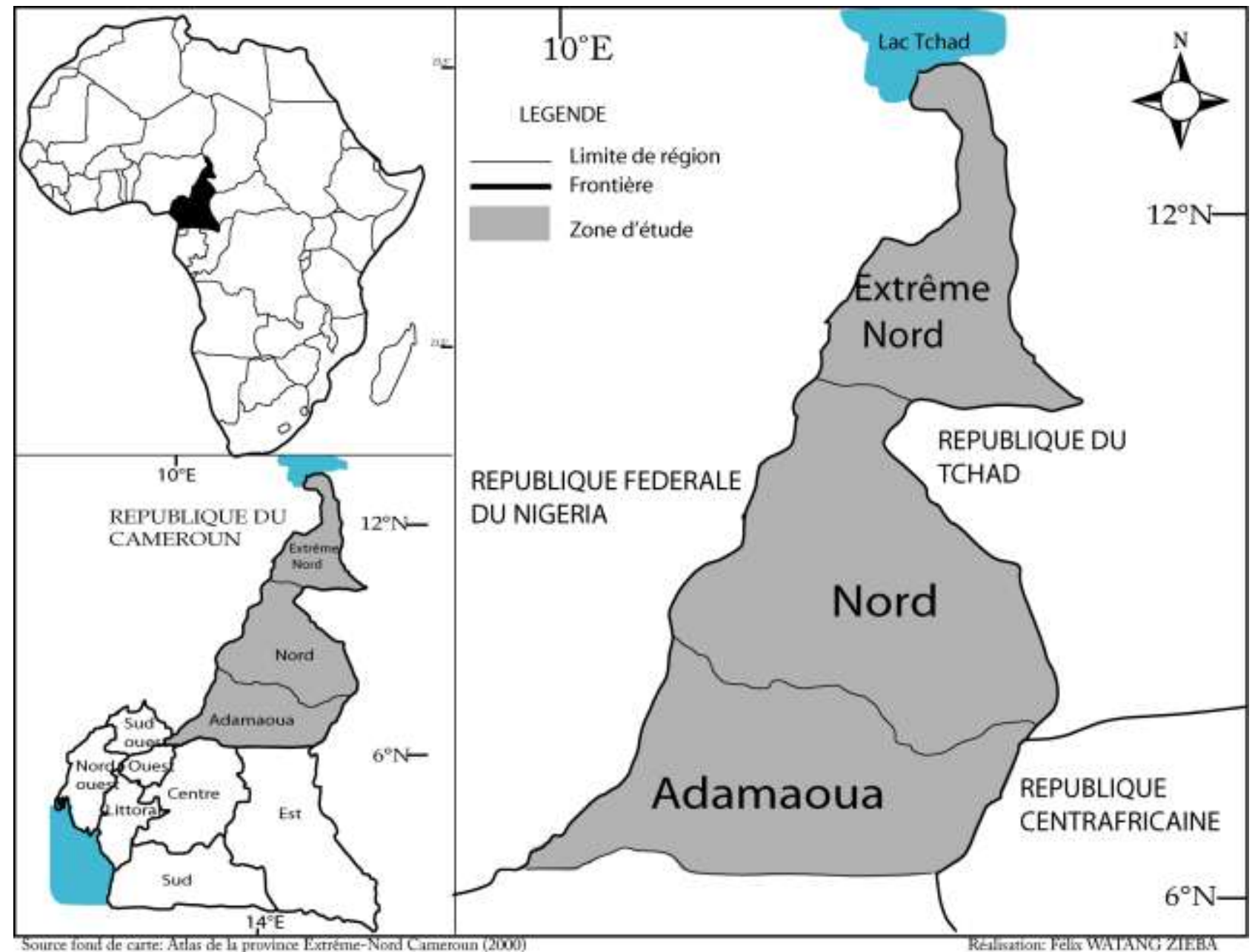

Figure I. Localisation de la zone d'étude

Notre stratégie de vérification de l'hypothèse selon laquelle, la mobilité croissante des populations rurales vers les centres urbains proches, l'immigration transfrontalière sont responsables du développement des villes du Nord Cameroun ces vingt dernières années, est l'observation documentaire appuyée par une observation de terrain.

\section{Les échelles d'observation}

Les échelles d'observations vont du quartier à la région septentrionale en passant par l'étalement urbain. En effet, l'observation à l'échelle du quartier nous a permise de lire la dynamique démographique liée à l'immigration dans ces unités plus faciles à enquêter.

L'observation à l'échelle de l'étalement urbain permet une lecture de la dynamique spatiale des villes étudiées. Nous avons insisté sur les villes de Maroua et Ngaoundéré.

L'observation à l'échelle régionale nous permet de montrer l'émergence de petits centres urbains à l'échelle de tout l'espace septentrional camerounais.

\section{La Collecte et l'analyse des données}

Les données collectées dans le cadre de cette études ont de types secondaires et primaires.

-Les données secondaires sont celles issues des recensements de la population et de l'habitat de 1976, 1987 et 2005. Les données de 2010 sont issues de la mise à jour des projections démographiques faites sur la base d'un scénario d'hypothèse forte à la suite du recensement général de la population et de l'habitat de 2005. Le critère démographique de 20000 habitants a été retenu pour identifier les villes lors de l'exploitation des données des différents recensements; ce qui nous permet de comparer le même phénomène d'un recensement à un autre.

-Les données primaires: les données secondaires ont été complétées par une enquête dans les quartiers "points de chute» et de résidence de migrants dans les principales villes du NordCameroun : Maroua, Garoua et Ngaoundéré ; dans les petites villes à forte immigration. Lesquels quartiers ont été préalablement identifiés à partir de l'exploitation des données secondaires (données de recensements antérieurs) et d'une descente 
exploratoire sur le terrain. La descente exploratoire sur le terrain se résume aux entretiens menés avec les chefs de quartiers qui ont donné les informations sur les différentes origines des habitants du quartier. Les enquêtes ont été réalisées grâce à un protocole articulé autour de l'identification de l'enquêté, du lieu d'origine, la date, les causes de l'immigration et des autres caractéristiques sociodémographiques.

L'analyse des données:

-La comparaison des données démographiques issues des différents recensements a permis d'apprécier l'émergence d'un nombre non négligeable de petites villes. II s'est agi aussi de calculer les pourcentages de croissance des villes d'un recensement à un autre. L'analyse statistique a permis de présenter l'évolution démographique des différentes villes sous forme de graphique et de tableau statistique.

-La cartographie a servi à la lecture et à l'analyse de l'évolution spatiale du phénomène urbain au Nord Cameroun. Grâce aux relevés GPS que nous avons effectués et projeter sur les fonds de carte numérique existantes, il a été possible de délimiter les extensions récentes des villes étudiées. La projection des coordonnées sur les cartes existantes permet de lire l'évolution spatiale des différentes villes.

\section{Résultats}

\section{La place des villes septentrionales dans l'urbanisation du Cameroun}

La hiérarchisation urbaine au Cameroun est dominée par les deux principales villes millionnaires que sont Douala et Yaoundé. Cette première classe est directement suivie par celle des villes ayant une population comprise entre 200000 et 300000 habitants. Les capitales régionales du Nord Cameroun appartiennent à cette deuxième classe (confère tableau $\mathrm{I}$ ).

\begin{tabular}{|l|l|l|}
\hline & Ville & Population \\
\hline I & Douala & I 907479 \\
\hline 2 & Yaoundé & I 817524 \\
\hline 3 & Bamenda & 269530 \\
\hline 4 & Bafoussam & 239287 \\
\hline 5 & Garoua & 235996 \\
\hline 6 & Maroua & 201371 \\
\hline 7 & Ngaoundéré & 152698 \\
\hline 8 & Kumba & 144268 \\
\hline 9 & Nkongsamba & 104050 \\
\hline 10 & Buea & 90088 \\
\hline
\end{tabular}

Source : Recensement Général de la population et de l'Habitat (RGPH), 2005

Tableau I. Classement des dix premières villes du Cameroun

Les trois capitales régionales du Nord Cameroun font partie des dix premières villes du Cameroun et deux d'entre elles font partie des quatre villes moyennes de plus de 200000 habitants (figure 2) 
Figure 2. Hiérarchisation urbaine au Cameroun

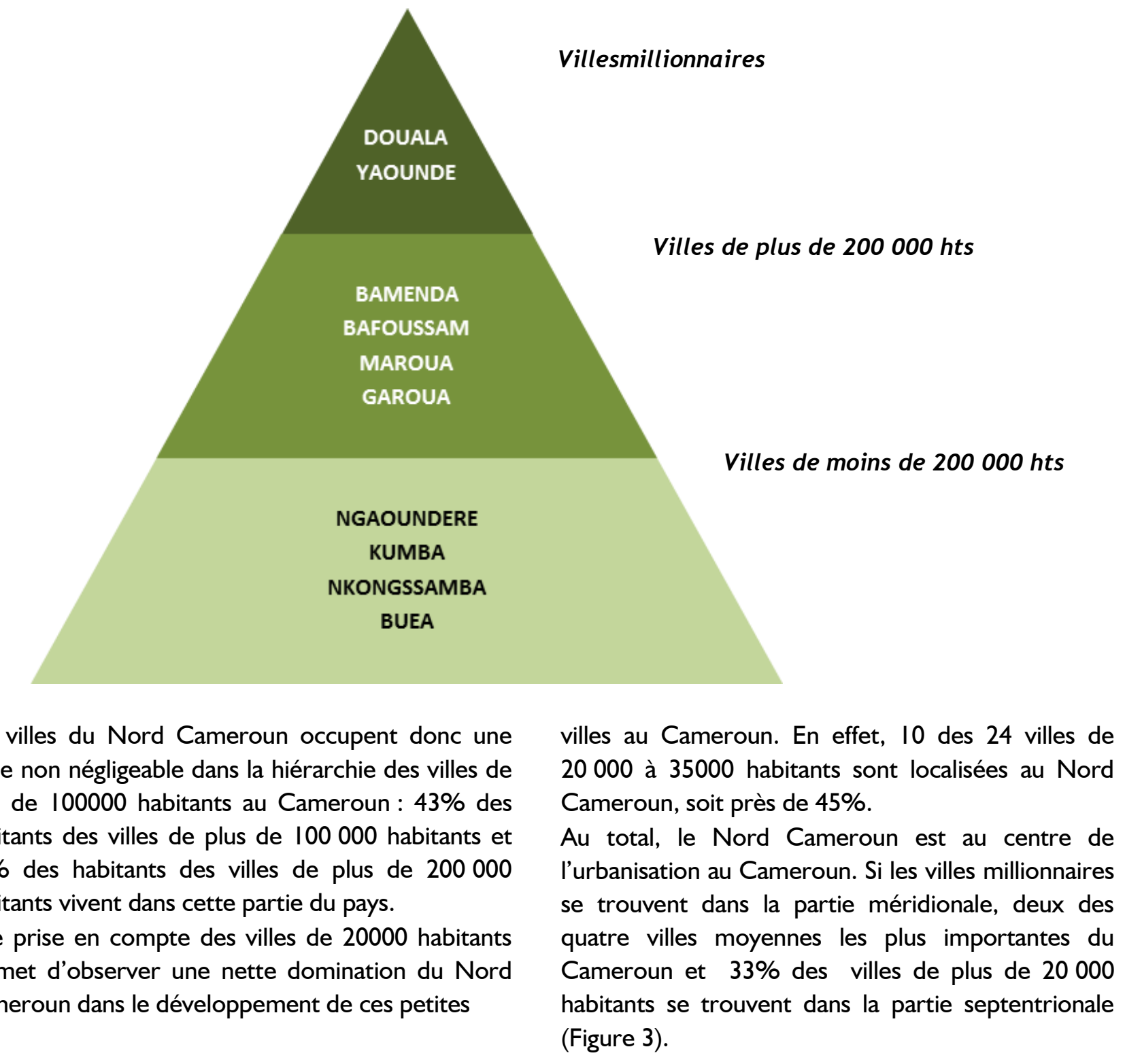

Les villes du Nord Cameroun occupent donc une place non négligeable dans la hiérarchie des villes de plus de 100000 habitants au Cameroun : $43 \%$ des habitants des villes de plus de 100000 habitants et $46 \%$ des habitants des villes de plus de 200000 habitants vivent dans cette partie du pays.

Une prise en compte des villes de 20000 habitants permet d'observer une nette domination du Nord Cameroun dans le développement de ces petites villes au Cameroun. En effet, 10 des 24 villes de 20000 à 35000 habitants sont localisées au Nord Cameroun, soit près de $45 \%$.

Au total, le Nord Cameroun est au centre de urbanisation au Cameroun. Si les villes millionnaires se trouvent dans la partie méridionale, deux des quatre villes moyennes les plus importantes du Cameroun et $33 \%$ des villes de plus de 20000 (Figure 3). 


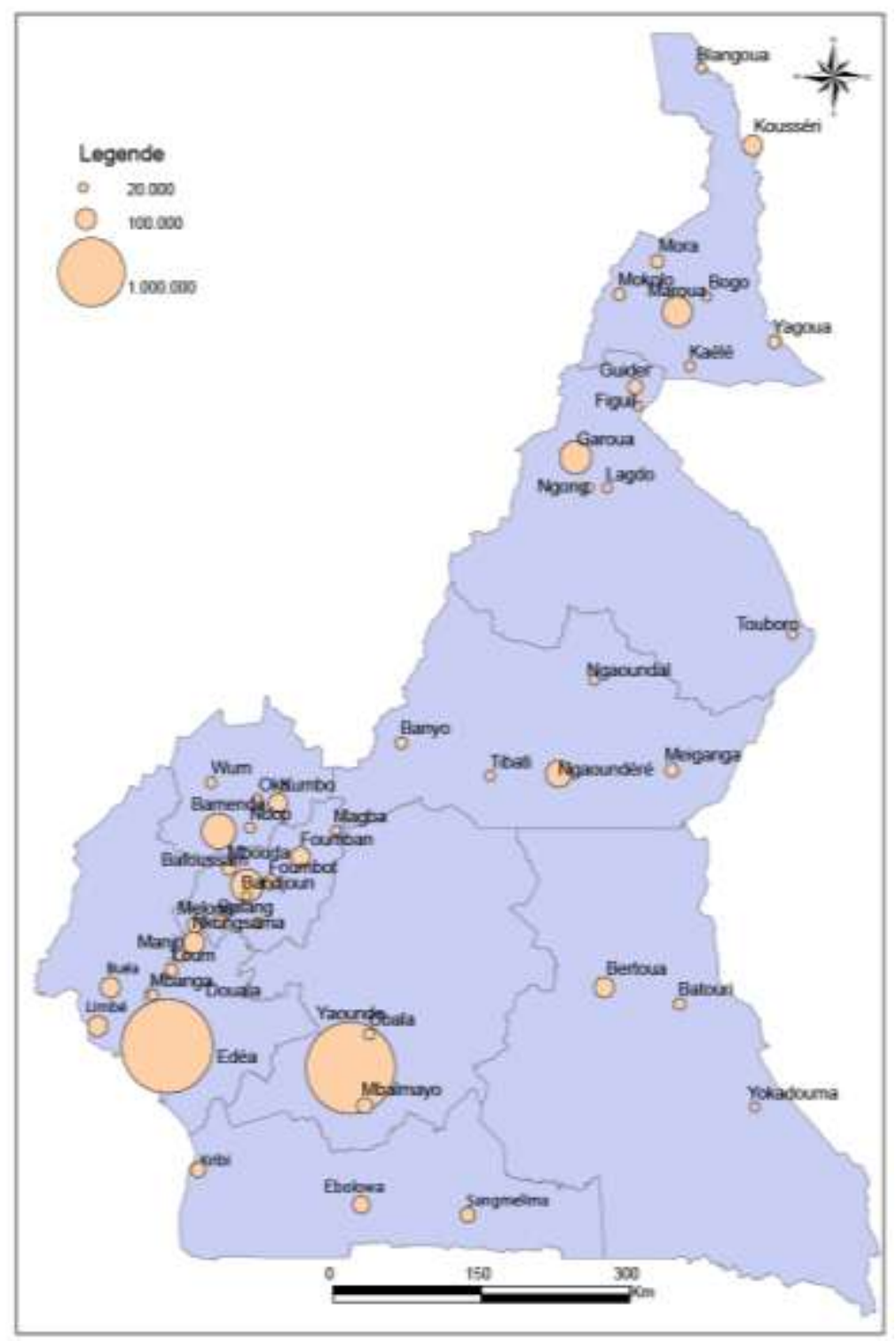

Source données statistiques: Recensement Général de la Population et de l'Habitat (RGPH), 2005

Figure 3. Villes de plus de 20000 habitants

Ces villes appartiennent à deux des neufs grandes zones de peuplement de l'Afrique centrale telles que reparties par Gazel et al., (20I0) : le couloir sahélien constitué de la bande sahélienne du Cameroun, de la RCA, du Tchad et de l'Erythrée; et le chemin oubanguien passant par le Cameroun, la RCA, le Tchad et le Soudan) dont les taux d'urbanisation ne dépassent pas $20 \%$ de la population totale.

\section{L'immigration comme facteur de la}

\section{dynamique urbaine au Nord Cameroun}

Les chefs-lieux d'administration furent longtemps considérés comme les principaux centres urbains du Nord Cameroun. La hiérarchisation administrative correspondait alors dans ce contexte à la hiérarchisation urbaine. L'accélération de la dynamique urbaine va modifier ce schéma à travers l'émergence de quelques centres (Figuil, Guider, Kousséri, Lagdo, Ngong) qui bénéficient d'un afflux de migrants plus importants (RGPH, 1987 et 2005).

Mobilités spatiales, immigration transfrontalière et dynamique des trois capitales régionales : Maroua, Garoua, Ngaoundéré

Les capitales des trois régions administratives de la partie septentrionale du Cameroun constituent aussi les principales villes de cette partie du pays. Ces villes connaissent depuis plus de vingt ans déjà une croissance démographique continue (figure 4) 
Source: Recensemen tGénéral de la Population et de l'Habitat (RGPH), 1976, 1987, 2005

Figure 4. Evolution de la population des trois métropoles régionales du Nord Cameroun entre 1976 et 2005

Les villes de Maroua, Garoua et Ngaoundéré connaissent une évolution régulière depuis 1987. Ces trois villes constituent un foyer de peuplement accueillant des populations d'origine diverse. Elles participent à l'organisation de leurs espaces régionaux respectifs et constituent une zone de convergence des ruraux de la région.

- Migrations internes et dynamique démographique des capitales régionales
La population des capitales régionales du Nord Cameroun a doublé entre 1987 et 2005 ( figure 2). Elle est passée de 123296 à 201376 habitants pour la ville de Maroua et de 141839 à 235996 habitants pour la ville de Garoua ; soit un taux de croissance annuel moyen de 3,5\% pour la ville de Maroua et de $3,6 \%$ pour la ville de Garoua correspondant à la moyenne nationale. Les migrations internes en général et l'exode rural en particulier participent de façon significative à cette croissance (figure 5).

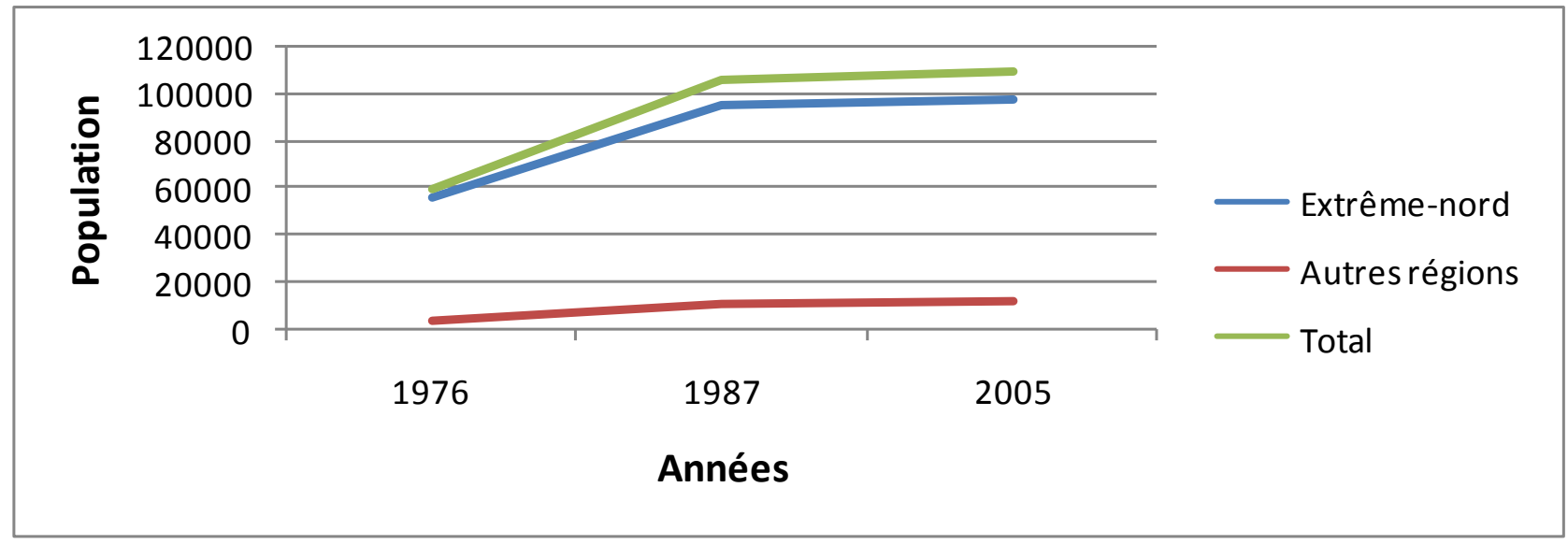

Source: RecensementGénéral de la Population et de l'Habitat (RGPH), 1976, 1987, 2005

Figure 5. Evolution de la population nationale immigrée à Maroua selon le lieu d'origine (1976-2005) 
L'exode rural constitue le principal facteur de croissance démographique dans la ville de Maroua. Plus de 100000 habitants de la ville de Maroua viennent de la région environnante (Extrême-Nord). Les principales zones de départ sont les monts Mandara (lyébi-Mandjek, 1997), la plaine de Kaélé et la vallée du Logone. On note aussi une immigration des populations venant des autres régions du Cameroun (près de I 2000 habitants). II s'agit en fait des commerçants, des fonctionnaires et depuis 2008 des étudiants originaires des régions méridionales. L'immigration transfrontalière occupe aussi une place non négligeable dans la croissance démographique.

- Migrations dynamique principaux Cameroun

\section{transfrontalières et démographique des centres urbains du Nord}

L'immigration transfrontalière relève pour la plupart des tchadiens. II s'agit d'une part de riches immigrés

\begin{tabular}{|l|l|l|l|l|}
\hline Villes & Quartiers & Effectif enquêté & Population étrangère & Nationaux \\
\hline \multirow{2}{*}{ Maroua } & Pont vert & 100 & 21 & 79 \\
\cline { 2 - 5 } & Sararé & 100 & 38 & 62 \\
\hline Garoua & Roumdé Adjia & 100 & 29 & 71 \\
\hline Ngaoundéré & Joli soir & 100 & 21 & 79 \\
\hline Total & $\mathbf{4 0 0}$ & 109 & 291 \\
\hline
\end{tabular}

Source: enquête de terrain

Tableau 2. Population étrangère dans quelques quartiers des principales villes du Nord Cameroun

- Dynamique spatiale des villes de Maroua, Garoua, Ngaoundéré (figure)

L'extension urbaine et la spéculation foncière résultent de l'immigration sans cesse croissante dans les villes du Nord Cameroun. L'installation des vagues successives d'immigrés venus des campagnes proches a favorisé le développement des quartiers périphériques qui se propriétaires de résidence secondaires où vivent leurs familles et d'autre part des populations pauvres originaires du sud ouest tchadien à la recherche d'un emploi ou ayant fui la guerre. A ces immigrés tchadiens, il faut ajouter les artisans et commerçants nigérians, nigériens, ghanéens, maliens et sénégalais. A Maroua, la population étrangère est évaluée à 1472 individus (RGPH 2005). Cependant, si on considère comme critère de définition du migrant tchadien, le lieu de naissance de l'individu et de ses parents au Tchad, il est clair que ce chiffre devrait être multiplié au moins par trois. Car d'après les enquêtes menées à Maroua, Garoua et Ngaoundéré (tableau 2), près de $40 \%$ des enquêtés considérés dans le cadre de cette étude comme migrants ont une carte nationale d'identité camerounaise. Ce qui les exclue officiellement de la catégorie tchadienne. Celle-ci constitue parfois près de $40 \%$ de l'effectif de certains quartiers (tableau 2)

sont étendus sur les zones non aedificandi (zones inondables, flancs de montagne). C'est le cas des quartiers Louggéo, Djoudandou à Maroua, Houro ngalbidjé à Garoua, Socaret II, Jérusalem à Ngaoundéré.

Une enquête menée dans le secteur I du quartier Socaret II à Ngaoundéré auprès de 100 ménages tirés au hasard sur les 298, révèle les résultats suivants : 


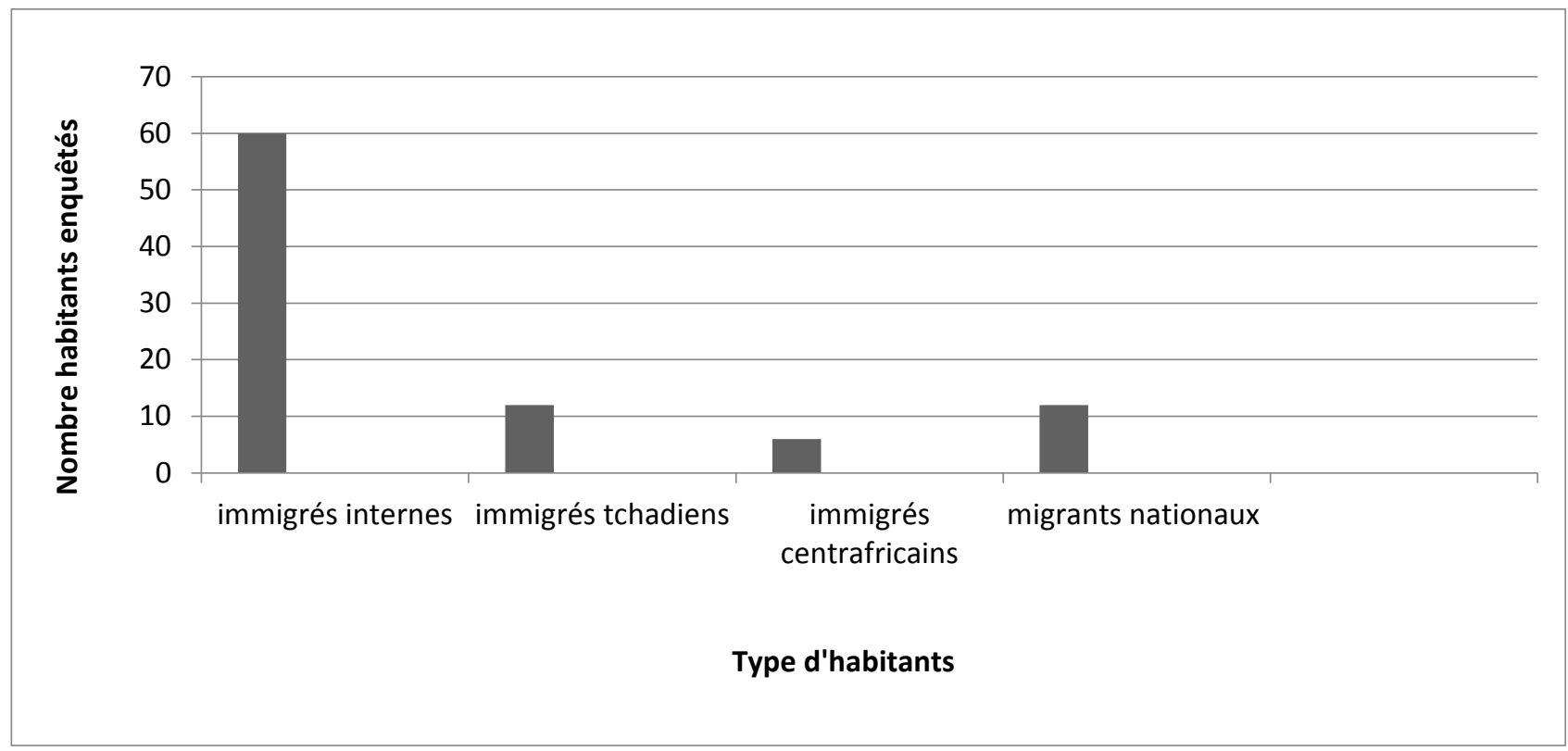

Source: Enquête de terrain, 2012

Figure 6. Proportion de la population immigrée dans un quartier périphérique de Ngaoundéré

Le cas du quartier Socarect II illustre bien l'extension des villes suite à l'immigration interne et transfrontalière. Tel est aussi le cas pour les villes de Garoua et Maroua.

A ceci s'ajoute le développement des quartiers de résidences secondaires construites par les migrants nantis. L'arrivée de ces migrants constitue l'une des vagues les plus récentes de l'immigration transfrontalière. Elle date des années 2000 après le boom pétrolier tchadien. L'émergence d'une classe de riches tchadiens ayant les moyens d'acquérir une propriété secondaire sera suivie par le développement du tourisme dans les villes proches du Tchad (Kousséri, Maroua).
Ces mêmes villes serviront plus tard de zone de replis ou de refuges lors des troubles politiques de la fin de cette décennie. Les refugiés tchadiens avec résidence (refuges) secondaires sont des citoyens tchadiens qui disposent des moyens d'accès à une propriété immobilière dans les différentes villes du Nord Cameroun. II s'agit des villes de Kousséri, Maroua, Garoua et Ngaoundéré. Certaines de ces habitations sont occupées seulement le week-end et durant les périodes d'instabilité à N'Djamena comme ce fut le cas en 2008'. Ces villas cossues sont occupées pour la plupart de temps par des vigiles.
'Affrontements armés les 2 et 3 février 2008 entre des forces rebelles et l'armée nationale tchadienne à Ndjamena 


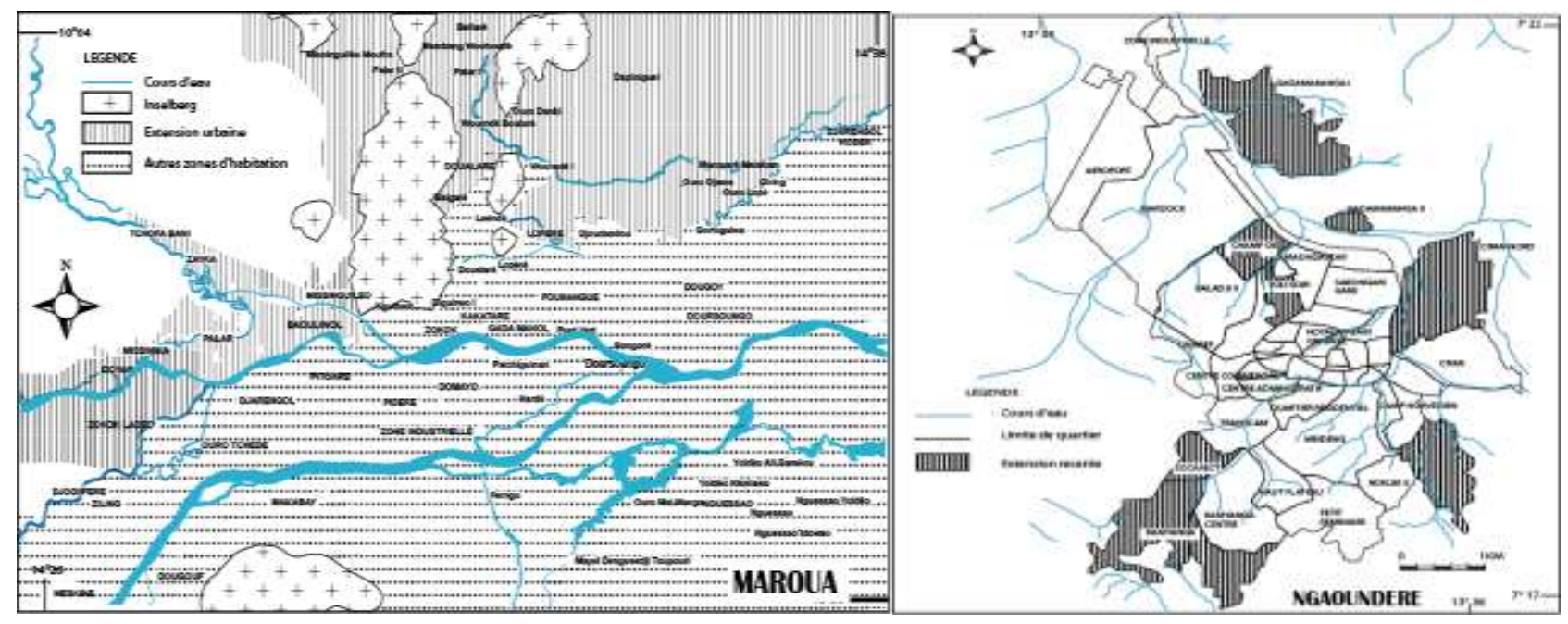

Source: Levés de terrain, 20I I-2012

Figure 7. Extension spatiale des villes de Maroua et Ngaoundéré

Certaines habitations sont occupées en permanence par les familles des propriétaires vivant au Tchad. C'est le cas des quartiers Champ de prière et Baladji II à Ngaoundéré, Dougoï et Baoléol à Maroua qui enregistrent une forte présence tchadienne. La sécurité et l'éducation de qualité dans les collèges de renom (Collèges de Mazenod, Protestant et AMYTI de Ngaoundéré, Collège Sainte Thérèse de Garoua, Collège Jacques de Bernon de Maroua) justifient l'installation régulière du reste de la famille.

D'autres villes secondaires connaissent aussi la même dynamique. II s'agit des petites villes commerciales et frontalières.

\section{Dynamique des petites villes commerciales et frontalières : Kousséri, Guider, Figuil}

Kousséri et Guider ont respectivement une population de 89123 et 52316 habitants (RGPH, 2005) (figure 3). Elles font partie des cinq villes du Nord Cameroun ayant une population de plus de 50000 habitants. La ville de Kousséri a enregistré entre 1976 et 1987, le taux d'accroissement annuel le plus élevé et jamais enregistré du Cameroun, soit 14,2\%. Ces petites villes constituent un second pôle de convergence des populations. 


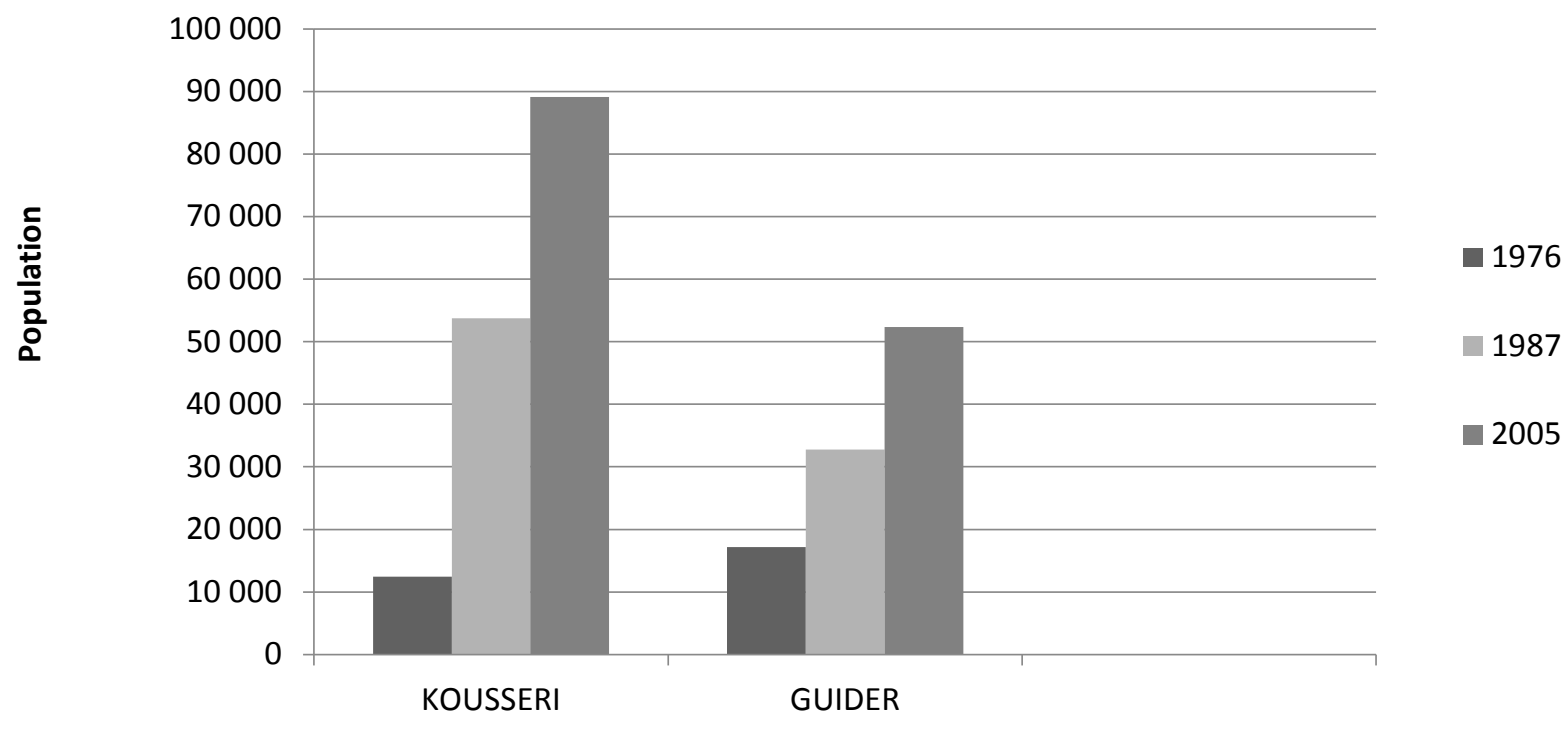

Villes

Source: Recensement Général de la Population et de l'Habitat (RGPH), 1976, 1987, 2005

Figure 8. Evolution de la population dans les villes de Kousséri et de Guider entre 1976 et 2005

Les villes de Kousséri, Figuil et Guider sont des villes frontalières au Tchad (figure 6). Elles entretiennent de fortes relations commerciales avec les villes tchadiennes et leurs régions: N'Djaména pour Kousséri, Léré et le sud ouest tchadien pour Figuil et Guider. Ces villes entretiennent aussi des relations avec le Nigéria : c'est le cas de Guider avec la ville de Mobi au Nigéria, de Kousséri avec la ville nigériane de Maïduguri.

La proximité géographique de ces villes avec le Tchad font d'elles des pôles d'attraction et des zones de refuge pour les populations tchadiennes. II est difficile de dénombrer la population tchadienne vivant dans ces villes à cause de l'irrégularité des mouvements migratoires. Une enquête sur le lieu de naissance des parents d'élève à été menée auprès de 300 élèves du Cours Moyen première année (CMI) repartis dans six écoles primaires (par ville, les deux écoles ayant les plus grands effectifs ont été retenues) choisies au hasard dans les villes de Guider, Kousséri, Figuil. Un échantillon de 50 élèves sur une population de 90 par classe a été défini.) Les résultats de l'enquête montrent que de nombreuses familles d'origine tchadienne sont installées durablement dans ces villes (tableau 3).

\begin{tabular}{|l|c|c|c|}
\hline Villes & $\begin{array}{l}\text { Effectif } \\
\text { enquêté }\end{array}$ & $\begin{array}{c}\text { Elèves d'origine } \\
\text { tchadienne }\end{array}$ & Autres \\
\hline Figuil & 100 & 21 & 79 \\
\hline Guider & 100 & 13 & 87 \\
\hline Kousséri & 100 & 31 & 69 \\
\hline Total & $\mathbf{3 0 0}$ & $\mathbf{6 5}$ & $\mathbf{2 3 5}$ \\
\hline
\end{tabular}

Source : enquête de terrain, 20II

Tableau 3. Proportion des élèves étrangers dans les écoles enquêtées à Figuil, Guider, Kousséri

Plus de $21 \%$ d'écoliers enquêtés dans des écoles choisies de façon aléatoire dans les villes de Kousséri, Guider et Figuil sont d'origine étrangère. Ceci témoigne de l'apport de l'immigration dans la dynamique démographique.

II existe un troisième pôle urbain constitué de petites villes agricoles.

\section{Le développement de petites villes agricoles : Maga, Ngong-Tchéboa, Lagdo, Touboro, Pitoa}

Ce sont des villes qui se sont développées dans les pôles agricoles du Nord Cameroun : la vallée rizicole du Logone et la vallée de la Bénoué. Les vallées du Logone (Extrême-Nord) et de la Bénoué (région du Nord) ont bénéficié des aménagements agricoles depuis les années 1970. La croissance de la production agricole qui en résulta a favorisé le 
développement des marchés de collecte et autres activités commerciales. Plus de 200000 migrants internes se sont installés dans ces pôles agricoles (Roupsard, 1987). Les statistiques relatives aux immigrés transfrontaliers sont difficiles à obtenir à cause de la spontanéité de ce mouvement migratoire.

Le fait que Maga, Ngong, Lagdo soient des villes créées par des immigrés, confirme le rôle de l'immigration dans l'urbanisation de ces bassins agricoles. Ce sont de véritables villes agricoles dont la population est supérieure à celles de certains chefs lieux de département; le critère administratif étant considéré au Cameroun comme essentiel à la définition de la ville.

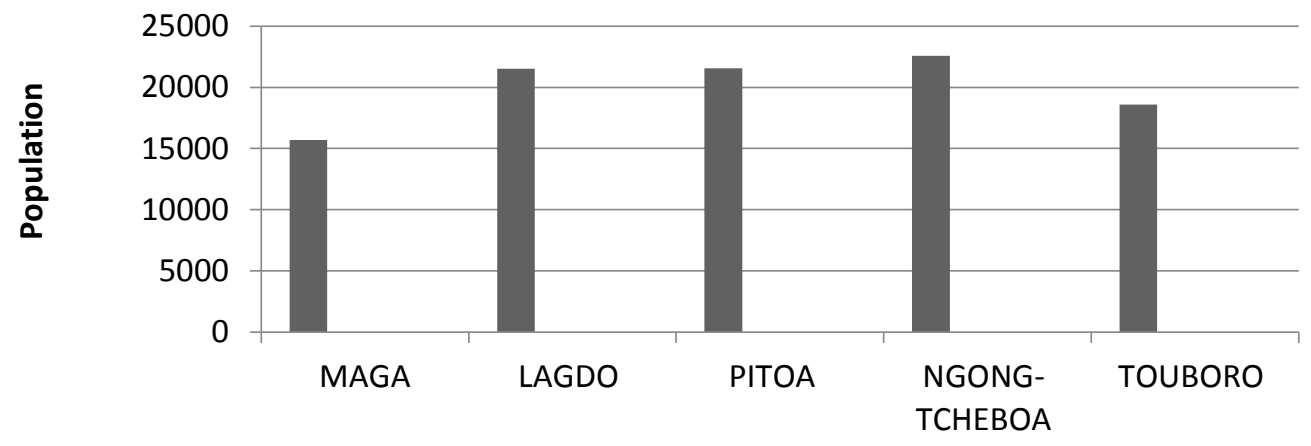

VILLES

Source: Recensement Général de la Population et de l'Habitat (RGPH), 2005

Figure 9. Population des villes de Maga, Lagdo, Pitoa, Ngong-Tchéboa, Touboro

Ces petites villes agricoles ont une population moyenne de 20000 habitants.

\section{Discussion}

La dynamique migratoire accélère l'urbanisation au Nord Cameroun depuis la fin des années 1980. D'espace d'émigration alimentant le pôle urbain méridional (Douala, Yaoundé), cette partie du Cameroun est devenue un bassin d'immigration internationale (tchadiens, nigérians, nigériens, maliens, centrafricains) et interne. Autour des trois capitales régionales (Maroua, Garoua et Ngaoundéré) qui connaissent une croissance spatiodémographique importante, émergent des villes moyennes commerciales et des petites villes agricoles de plus de 20000 habitants. Les villes moyennes contribuent ainsi à l'évolution urbaine de l'hinterland camerounais et offrent des opportunités pour des transformations socioéconomiques.

Les données issues des recensements nationaux de 1976, 1987 et 2005 peu précises sur les questions migratoires ont été complétées par des enquêtes de terrain à l'échelle des quartiers méthodiquement sélectionnés. Ces données secondaires parfois douteuses en ce qui concerne l'immigration internationale, méritent d'être questionnées et confrontées sur le terrain. En effet, les opérations de recensement ne peuvent prendre en compte des réalités sociopolitiques qui permettent au migrant de se fondre dans la société d'immigration. II s'agit notamment des cartes nationales d'identité parfois obtenues dans des conditions douteuses lors des campagnes d'établissement gratuit desdites cartes.

L'analyse des données secondaires et de terrain ont permis de vérifier la relation entre immigration et urbanisation au Nord Cameroun et démontre l'extension du pôle urbain septentrional. Ceci confirme la dynamique urbaine à l'échelle continentale relevée par Eric-Denis et MoriconiEbrard (2009). 


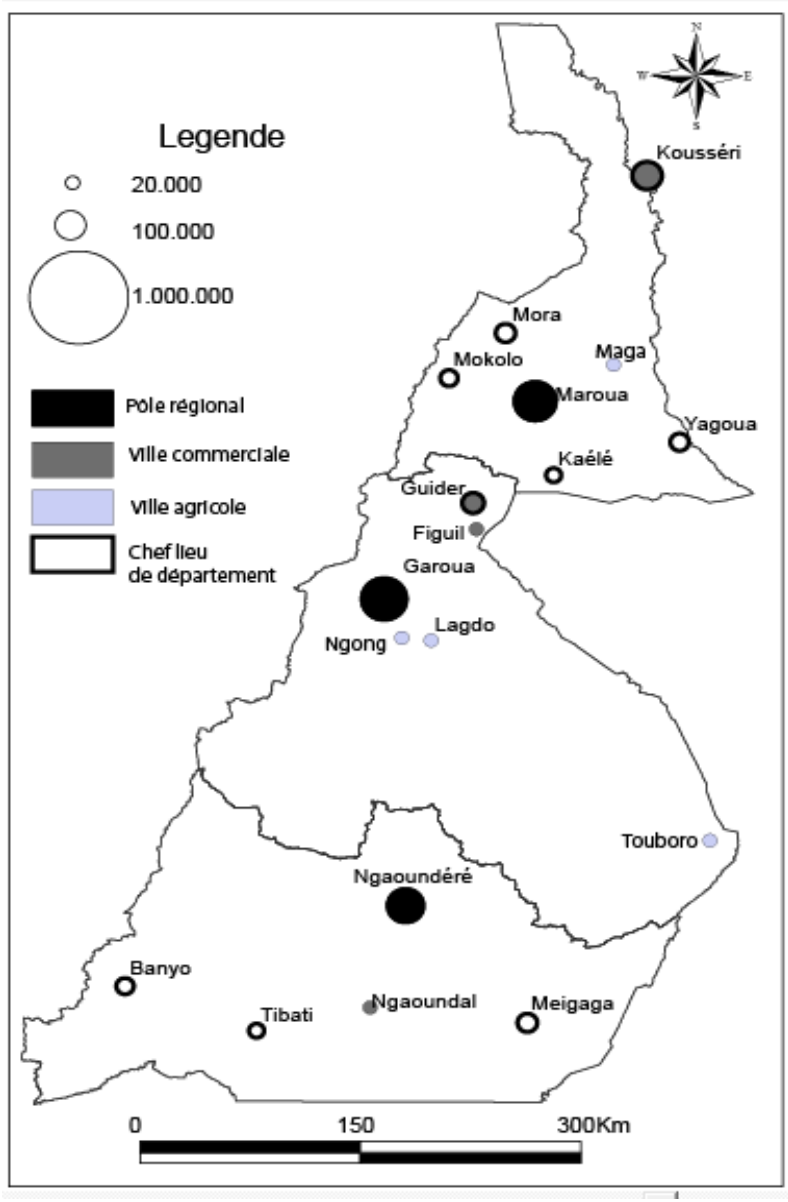

Figure 10. Localisation des foyers de peuplements urbains au Nord Cameroun

\section{Conclusion}

L'exode rural et l'immigration transfrontalière expliquent en partie l'urbanisation au Nord Cameroun ces vingt dernières années. L'analyse statistique des données résultant des enquêtes auprès des habitants des villes du Nord Cameroun et les autres observations de terrain permettent de mettre en relation l'immigration, la croissance démographique et la dynamique spatio-économique des principales et petites villes du Nord Cameroun. II apparait que le Nord Cameroun constitue un pôle important de développement de villes moyennes de plus de 200000 habitants et de petites villes de 20000 habitants.

L'extension spatiale est le résultat de la mise en valeur des espaces périphériques par les immigrés en quête d'espace de construction. L'entrée en jeu de nouveaux acteurs économiques notamment des commerçants et petits industriels chinois, la diversification de l'activité économique, le développement des établissements bancaires viennent marqués l'amorce d'une dynamique économique nouvelle. Le Nord Cameroun apparaît alors comme un espace en cours d'urbanisation et comme une future région urbaine du Cameroun. En effet, la connectivité entre les grandes villes de cette partie du Cameroun (Maroua, Garoua et Ngaoundéré) d'une part, entre les grandes villes et petites villes commerciales et agricoles d'autre part est réelle.

\section{Bibliographie}

Beauchemin, C., 2005, "Pour une relecture des tendances migratoires entre villes et campagnes: une étude comparée Burkina Faso - Côte d'lvoire» in Études de la Population Africaine (20) I, pp. I4I-I65.

Beauchemin C., Schoumaker B., 2005, "Migration to Cities in Burkina Faso: Does the Level of Development in Sending Areas Matter?" in World Development, Vol. 33, No. 7, p. II29-II 52 Beauchemin C., Bocquier Ph., 2004, "Migration and Urbanization in Francophone West Africa: An Overview of the Recent Empirical Evidence", in Urban Studies, Vol. 4I, No I I, pp. 2245-2272

Courade, G., (sous la direction de), 1994, Le village camerounais à l'heure de l'ajustement, KarthalaParis, (Economieetdéveloppement), 392p.

Eric-Denis, Moriconi-Ebrard, F., 2009, Dynamique de l'urbanisation, 1950-2020: Approche géostatistique, Afrique de l'ouest, Agence Française de Développement (AFD) / e-Geopolis, 123p.

Gazel, H., Harre, D., Moriconi-Ebrard, F., 2010, Le Rapport général de l'étude "Africapolis II, L'urbanisation en Afrique centrale et orientale ", Agence Française de Développement (AFD) / eGeopolis, 20p.

Dongmo, J-L, 1980, "Polarisation de l'espace camerounais : les champs migratoires des villes. ", in Revue de Géographie du Cameroun, Yaoundé vol I, n², pp I45-160

Gonné B., 2009, "Les Migrations saisonnières transfrontalières de la main d'œuvre agricole tchadienne à l'Extrême-Nord du Cameroun » in Kaliao, série sciences humaines, revue de I'ENS/Université de Maroua (Cameroun), voll

Guilmoto, C., Sandron, F., 2000, Migration et développement, Paris, La documentaion française, 2003, I44p.

Harris, J.R. etTodaro, M.P., 1970, "Migration, unemployment and development: $\mathrm{A}$ two-sector Analysis", in American economic review, vol. 60, pp |26-I4I

Hoyaux, A-F., 2010, "La penséegéographique de la ville et de l'urbain en France" in Laurent Cailly et Martin Vanier (Ed.), La France : 
UneGéographieUrbaine , Armand Colin, Collection U Géographie, 365 pages

lyebi-Mandjek, O., 1993, "Les migrations saisonnières chez les Mafa, montagnards du Nord Cameroun : une solution au surpeuplement et un frein à l'émigration définitive. ", in Cahiers des sciences humaines, vol. 29, $\mathrm{n}^{\circ} 2-3$, Editions de l'ORSTOM, Paris, pp4l9-437.

lyebi-Mandjek, O., 2005, «typologie des mouvements migratoires "in XIII ${ }^{\text {ème }}$ Colloque International du Réseau Méga-Tchad, Migrations et mobilités dans le bassin du lac Tchad, Maroua, 3 loctobre - 2 novembre 2005, IRAD-IRD, Maroua, communication.

Kossoumna, Liba'a N., Havard, M., 2006, “Mutations de la filièrecotonnièredans les provinces septentrionales du Cameroun: perception etstratégiespaysannes " inCahiers de géographie du Québec, Volume 50, numéro 139, avril 2006, p. $65-82$

Kuby et al., 200I "Newton's first law of migration : "the gravity model.», pp87-108 inKuby M., Harner J., et Cober P., (Ed.), Human geography in action, John Wiley \& sons, inc, 438p.

Le Bris, E., 200I, "Croissanceurbaine et gestion des villes”, inLeryA., et Vimard P., (coordonné par), 200I, Population et développement : les principaux enjeux cinq ans après la conférence du Caire, PP36-42.

Marguerat Y., 1974, Migrations vers les villes et polarisationrégionale :l'exemple du Cameroun, Paris: ORSTOM, 1974, (39), p. I75-182.

Noin, D. etThumerelle, P-J., 1995 L'étudegéographique des populations, Masson Géographie, 126p

ONU, 2010, World Urbanization Prospects, The 2009 Revision, New York

Park, T., Greenberg J., Nell E., Marsh S., MamadouBaro, MouradMjahed, 2003, "Research on Urbanization in the Developing World: New Directions" in Journal of Political Ecology Vol. 10 , pp69-94

Pierre George, Verger F., 1996, Dictionnaire de la Géographie, $6^{\text {ème }}$ édition, PUF, $510 \mathrm{p}$
RGPH, 2005, Rapport de présentation des résultats définitifs, BUCREP (Bureau Central des Recensements et des Etudes de Population), 67p.

Roupsard, M., 1987, Nord Cameroun : Ouverture et développement. Impression et façonnade : Claude Bellée, Coutances, Manche, France, $516 \mathrm{p}$.

Saibou, Issa, 1997, "L'impact socio économique du séjour des réfugiés tchadiens à Kousseri (1979. 1982) " in NgaoundéréAnthropos, revue des sciences sociales, vol 2, pp I27-I37

Seignobos, CH. etlyebi-Mandjek, O., 2000, Atlas de la Province Extrême Nord du Cameroun, MINREST/INC/IRD, 84p. [Support numérique]

Simeu, Kamdem, 1985, Garoua, une métropole régionale au Cameroun. Thèse doctorat de $3 \mathrm{e}$ cycle, Université de Yaoundé, $437 \mathrm{p}$.

Simeu, Kamdem, 2000, La production urbaine au Cameroun Septentrional. ThèseDoctoratd'Etat, Université de Yaoundé, $737 \mathrm{p}$

Todaro, M.P, 1969, "A model of labor migration and urban unemployment in less developed countries" in American economic review, vol 59, mars, pp I38-I48

UNFPA, 2012, Etat de la population mondiale en 20II, 127p

Wackermann G., 2000, Géographie urbaine, Ellipses, Paris, 235p.

Chaline C., 1983, La dynamique urbaine, Paris, Ellipses, 200p.

Watang, Ziéba, F., 201I, "Immigration, croissancedémographique et dynamiqueurbaine au Nord Cameroun", communicationprésentéeà la Sixième Conférence Africaine sur la Population, Population africaine: passé, present et futur, Ouagadougou, Burkina Faso, 5-9 décembre 20I I

Zoa Zoa Y., 2010, Les migrations interterritoriales des plaines du Logone vers le bassin sucrier de Mbandjock-Nkoteng dans le Cameroun central : des impératifs socio-économiques à l'intégration nationale, Thèse de Doctorat de Géographie, Université de Bordeaux 